\title{
Evaluation of Genetic Variation in Local and Introduced Desert Banana (Musa sp.) Genotypes for Morpho-physicochemical Traits
}

\author{
Wassu Mohammed $^{1 *}$, Kebede W/Tsadik ${ }^{1}$, Tekalign Tsgaw ${ }^{1}$ and Kiflemariam Yehula ${ }^{2}$ \\ ${ }^{1}$ School of Plant Sciences, Haramaya University, P.O. Box: 138, Dire Dawa, Ethiopia \\ ${ }^{2}$ College of Agriculture, Bahirdar University, P.O. Box: 79, Bahirdar, Ethiopia
}

\begin{tabular}{|c|c|}
\hline Abstract & Article Information \\
\hline \multirow{14}{*}{$\begin{array}{l}\text { Knowledge of nature and magnitude of variation existing in available plant breeding materials } \\
\text { is of great importance for successful selection of varieties for yield and other desirable traits. } \\
\text { Therefore, this study was conducted to evaluate the genetic variation among } 4 \text { local and } 11 \\
\text { introduced desert banana (Musa sp.) genotypes. The genotypes were planted at Dire Dawa, } \\
\text { Eastern Ethiopia in randomized complete block design with five replications. Genetic variability } \\
\text { components analyses were conducted considering } 20 \text { morpho-physicochemical traits. The } \\
\text { results showed the presence of significant differences among genotypes for all traits. } \\
\text { Phenotypic and genotypic coefficient of variations ranged from } 8.95 \text { to } 52.63 \% \text { and } 7.2 \text { to } \\
48.16 \% \text {, respectively, with low magnitude of differences and moderate to high for most of the } \\
\text { traits. Heritability }\left(\mathrm{H}_{2}\right) \text { and genetic gain (GA) values were ranged from } 14.69 \text { to } 98 \text { and } 7.4 \text { to } \\
81.45 \% \text {, respectively, and both } \mathrm{H}_{2} \text { and GA values were high and moderate for } 16 \text { traits. Fruit } \\
\text { yield showed strong genotypic and phenotypic correlations with all growth traits and yield } \\
\text { components with higher magnitude of genotypic correlation coefficients. Euclidean distance } \\
\text { ranged from } 2.36 \text { to } 7.6 \text { which distinctly grouped genotypes into two clusters and five sub- } \\
\text { groups. Moreover, the local clones were more distant each other and with introduced } \\
\text { genotypes and performed better than introduced genotypes for most of the traits including fruit } \\
\text { yield. The study revealed the presence of genetic variation among local and introduced } \\
\text { genotypes and most of the traits were controlled more of by genetic factors. This suggested } \\
\text { the importance of exploiting genetic variability of local banana clones as equal to introduction } \\
\text { and evaluation of exotic genotypes and the higher chance of increasing fruit yield through } \\
\text { indirect selection for growth traits and yield components. }\end{array}$} & Article History: \\
\hline & Received : 18-09-2014 \\
\hline & : 05-12-2014 \\
\hline & $\begin{array}{l}\text { Accepted : 19-12-2014 } \\
\text { Keywords: }\end{array}$ \\
\hline & Cluster \\
\hline & Euclidean distance \\
\hline & Genetic gain \\
\hline & Genotypic and phenotypic \\
\hline & Variation \\
\hline & \\
\hline & ${ }^{*}$ Corresponding Author: \\
\hline & Wassu Mohammed \\
\hline & \\
\hline & \\
\hline
\end{tabular}

\section{INTRODUCTION}

Banana is the cheapest source of carbohydrate and a good source of vitamins A, B, C and minerals, which other staple food crops such as cassava, potato and cereals are lacking. Banana is one of the important fruit crops used both as staple and dessert. In some East and Central African countries, banana is a staple or co-stable food. As a result these communities, unlike the cereal producing ones, do not suffer from food shortage. Similarly, in southern and south-western Ethiopia, though population density is considerably high, people are not seriously affected by drought as those in northern and eastern Ethiopia mainly due to the use of enset (a relative of banana), banana, roots and tubers (EARO, 1999).

In Ethiopia, banana is produced throughout the country wherever there is adequate rainfall or irrigation opportunity. It is the second major fruit crop produced next to citrus. However, the research on banana was started in the country very recently (in early 1970s) as compared to other major crops. As a result few cultivars viz., Dwarf Cavendish, Poyo, Giant Cavendish, and Ducasse hybrid were recommended for production (EARO, 1999).
However, reports are lacking on the performance of local clones as compared to these introduced varieties. Cultivar development is a continuous activity which includes testing of improved varieties in many agro-ecologies and selection that can be made on local collections and/or introduced materials. In addition, in order to have a good choice of traits for selection of desirable genotypes, it is necessary to understand the nature and magnitude of variation existing in available plant breeding materials. It is also important to understand the interrelationships between quantitatively inherited plant traits for successful selection of varieties for yield (Khan et al., 2010; Kotal et al., 2010). But, in this regard, information is lacking for banana in the country.

Bananas are believed to have entered in the East African highland regions between the first and sixth century $A D$ (Price, 1995). As a result a wide range of unique varieties available that are belonging to the East African highland bananas and the region has been considered as secondary centre of Musa diversity (Swennen and Vuylsteke 1988; Stover and Simmonds 
Wassu Mohammed et al.,

1987). As part of this region, it is not far from truth to expect the presence of diversity among bananas of locally grown in Ethiopia, which might allow breeders to select clones for higher fruit yield and fruit quality. Therefore, germplasm characterization and classification provide useful information for the genetic betterment of the crop. Because knowing of the degree of genetic relatedness between clones and the range of diversity present in germplasm is important for conservation and selection of cultivars for diverse purposes in breeding programmes (Ortiz et al.., 1995; Garwel et al., 1992). Keeping in view this, the study was conducted to evaluate 11 introduced varieties of desert banana and four clones collected in eastern Ethiopia. As the part of the research project, genetic analysis was conducted with the objectives; i) to evaluate the genetic variation/distance among and between introduced and local desert banana genotypes and ii) to estimate phenotypic and genotypic variations and correlations, genetic advance and heritability of morpho-physicochemical traits.

\section{MATERIALS AND METHODS}

\section{Experimental Site, Design and Materials}

Fifteen desert banana genotypes were planted at Dire Dawa Agricultural Research Station of Haramaya University. Dire Dawa is located between $09^{\circ} 28.1 \mathrm{~N}$ and $41^{\circ} 38.1 \mathrm{E}$. The altitude of the station is 116 m.a.s.l. and the mean annual temperature ranges from 19 to $31.5^{\circ} \mathrm{C}$. The temperature is generally high with the monthly mean temperature of $28.1^{\circ} \mathrm{C}$ (December) and to $34.6{ }^{\circ} \mathrm{C}$ (June). Dire Dawa enjoys bimodal type of rainfall which is higher in April and July. The mean annual rain fall in the study area is $550 \mathrm{~mm}$ (Levoyageur Weather, 2012).

Eleven introduced desert banana clones namely; Kamaramesenge, Pissang Raja, Lactane, Butuza, Grande Naine, Poyo, Dwarf Cavendish, Giant Cavendish, Williams 2, Robusta, Williams 1 and four banana clones collected from eastern Ethiopia arbitrary named as Hararghe clone 1,2,3, and 4 were used for this study. Suckers of the banana clones were established in the field laid out in randomized complete block design (RCBD) with five replications. Five plants for each tested clone were planted in each replication with spacing of 2.5 meter between plants and 2.5 meter between rows. Irrigation, fertilization, weeding, sucker management were applied uniformly.

\section{Data Collection}

Morphological traits: Pseudostem height (PSH) was measured from the base of the plant to the top of the plant and girth of pseudostem (GS) was measured around the circumference using meter tape. Number of leaf per plant at harvest (NLH) was counted in each plant at the time of harvest while number of suckers until time of shooting (NSUTS) was recorded by counting the number of suckers produced by each plant up to shooting. Time interval between successive leaf emergences (TIBSLE) was recorded in days taking into consideration the emergence day of preceding leaf. Leaf length (LL) was measured from leaf blade base to the tip of the leaf and leaf breadth (LB) was measured at the point where the maximum breadth exists in the leaf at the time of flowering in each plant using tape meter.

Fruit Yield and Yield Components: Bunch weight (MBW) was measured using field balance after taking the
Sci. Technol. Arts Res. J., Oct-Dec 2014, 3(4): 19-28

bunch from the banana plant and number of hands per bunch $(\mathrm{MNH} / \mathrm{B})$ was recorded after counting hands in each bunch. Then after hands were separated from bunches and hands were weighted (MWH) in kilogram and fingers per hand $(\mathrm{MNF} / \mathrm{H})$ in each hand were counted. All fingers separated from a hand, weighted and divided to the number of fingers counted in each hand to obtain finger weight in grams (MWF), and fruit yield per hectare $(\mathrm{FY} / \mathrm{ha})$ was obtained by multiplying number of fruits produced in a hectare by the mean weight of fingers and converted to ton per hectare. Finger length (MF) and finger diameter (MFD) were measured in centimeter using ruler taking randomly selected 10 fingers from each bunch obtained from each plant in each replication.

Fruit Physicochemical Traits: Fruit peel (Mpel) and pulp (Mpulp) weights of fingers were measured in grams after the peel and pulp separated using sensitive balance. A $30 \mathrm{~g}$ of pulp was cut from fruits and diluted with $90 \mathrm{~g}$ of distilled water in a blender for one minute and filtered. A hand held refractometer was employed to measure the total soluble solid (TSS) in ${ }^{0}$ Brix where the reading was multiplied by three dilution factor. Titrable acid (TA) was measured from the diluted pulp titrated with $0.1 \mathrm{~N} \mathrm{NaOH}$ to the end point at $\mathrm{pH}=8.1$. Total soluble sugar to titrable acid ratio was obtained by dividing total soluble solid by titrable acid.

\section{Data Analysis}

Analysis of variance was computed for all traits considering randomized complete block design (RCBD). The phenotypic and genotypic variance and coefficient of variations were estimated according to the methods suggested by Burton and Devane (1953). Heritability $\left(\mathrm{H}_{2}\right)$ in broad sense was computed using the formula adopted by Allard (1960) and Falconer (1990) as: $H_{2}=\left[\sigma^{2} g / \sigma^{2} p\right] x$ 100 , where, $\sigma^{2} \mathrm{~g}=$ genotypic variance, $\sigma^{2} \mathrm{p}=$ phenotypic variance and $\sigma^{2} \mathrm{e}=$ error variance. Genetic gain as part of the mean (GA) for each trait was computed using the formula adopted by Allard (1960) and Johnson et al. (1955) as: Genetic gain $(\mathrm{GA})=(\mathrm{k})\left(\sigma^{2} \mathrm{p}\right)^{*}\left(\mathrm{H}_{2}\right)$, and expected genetic gain (EGA) (as \% of the mean) $=\left[\frac{(G A)}{X}\right] x 100$, where, $\mathrm{k}=$ selection differential $(\mathrm{k}=2.06$ at $10 \%$ selection intensity), $\sigma \mathrm{p}=$ phenotypic standard deviation, $\mathrm{H}_{2}=$ heritability in broad sense and $\mathrm{x}=$ grand mean. Phenotypic and genotypic correlation coefficients were computed using the method described by Miller et al. (1958).

Euclidean distance (ED) was computed from the 20 morpho-physicochemical traits of 15 desert banana genotypes/clones after standardization (subtracting the mean value and dividing it by the standard deviation) as:

$$
\mathrm{EDjk}={\sqrt{\sum_{i=1}^{n}(X i j-X i k)^{2}}}^{\frac{2}{2}} \quad \text { (Sneath and Sokal, 1973), }
$$

where EDjk = distance between clones $\mathrm{j}$ and $\mathrm{k}$; $\mathrm{xij}$ and $\mathrm{xik}=$ morpho-physicochemical traits values of the ith character for clones $\mathrm{j}$ and $\mathrm{k}$, respectively; and $\mathrm{n}=$ number of morpho-physicochemical traits used to calculate the distance. The distance matrix from morphophysicochemical traits was used to construct dendrograms based on the Unweighted Pair-group Method with Arithmetic means (UPGMA). The results of cluster analysis were presented in the form of dendrogram. In addition, mean ED was calculated for 
Wassu Mohammed et alo,

each clone by averaging of a particular clone to the other 14 clones.

Analysis of variance, phenotypic and genotypic variance, coefficient of variation and correlation were computed with SAS statistical software (9.0), heritability and genetic advance were computed using Excel Microsoft program. Dendrogram generated based on Unweighted Pair-group Method with Arithmetic means (UPGMA) and Euclidean distances depicting genetic relationships among 15 desert banana clones based on 20 morph-physicochemical traits were computed using STATISTICA-7 basic statistical analysis soft ware (2002).

\section{RESULTS}

\section{Analysis of Variance and Mean Performance of Genotypes}

Analysis of variance result for 20 morphophysicochemical traits in 15 desert banana genotypes is presented in Table 1. The result showed the presence of highly significant $(P<0.01)$ variation among genotypes for all traits except significant $(P<0.05)$ difference was observed for mean hand weight.

The mean values of 15 desert banana genotypes for 7 8 and 5 growth traits, fruit yield and yield components, and physicochemical traits are given in Table 2, Table 2, Table 3, and Table 4, respectively,. The overall mean values of local collections were greater than the introduced desert banana genotypes for all growth habits except for pseudostem girth (Table 2). The largest mean pseudostem height $(3.63 \mathrm{~cm})$ and pseudostem girth $(77.6$ $\mathrm{cm}$ ) were measured for introduced genotype, Pissang Raja while the largest time interval between successive leaf emergence (9.4) and highest number of suckers until time of shooting (10) were recorded for Kamaramesenge. Highest number of leaves (16) and leaf length $(168.4 \mathrm{~cm})$ at harvest were obtained from local Hararghe clone 2
Sci. Technol. Arts Res. J., Oct-Dec 2014, 3(4): 19-28

while the introduced genotype Williams 2 had the widest leaf $(67 \mathrm{~cm})$.

Local banana collections registered higher overall mean values for 8 out of 13 physicochemical traits including fruit yield tha while introduced genotypes exhibited higher overall mean values for mean hand weight, mean finger length, total soluble solid, titrable acid and total soluble solid to titrable acid ratio. However, when individual genotypes mean values were considered, only one local collection (Hararghe clone 2) recorded highest mean values for three traits and one local collection (Hararghe clone 1) registered second highest mean value for fruit yield and highest value for total soluble solid.

Genetic and Phenotypic Variation, Heritability and Genetic advance

Genetic variability estimates including genotypic and phenotypic variance, phenotypic (PCV) and genotypic (GCV) coefficient of variation, heritability, and genetic advance as percent mean for 20 morpho-physicochemical traits in desert banana genotypes are presented in Table 5.

The phenotypic variance was higher than the genotypic variance for all the traits studied. The magnitude of difference between phenotypic and genotypic variance were low for most of other traits. However, the higher magnitude of differences between phenotypic and genotypic variance as well as coefficient of variance were observed in mean hand weight, finger yield per hectare, mean bunch weight, number of suckers until time of shooting, total soluble sugar to titrable acid ratio, mean finger diameter, titrable acid, mean pulp weight, mean finger weight, mean number of hand per bunch and mean peel weight.

Table 1: Mean squares from analysis of variance for 20 morpho-physicochemical traits of 15 desert banana genotypes

\begin{tabular}{|c|c|c|c|c|}
\hline Trait & $\operatorname{Rep}(4)$ & Geno (14) & Error (56) & CV (\%) \\
\hline Mean pseudostem height $(\mathrm{m})$ & 165.97 & $3.73^{* *}$ & 0.03 & 9.39 \\
\hline Girth of pseudostem (cm) & 68.62 & $231.51^{* *}$ & 34.83 & 8.77 \\
\hline Time interval between successive leaf emergence & 1.38 & $7.96^{\star \star}$ & 1.08 & 12.69 \\
\hline Number of suckers until time of shooting & 2.55 & $11.97^{* *}$ & 3.23 & 27.62 \\
\hline Mean number of leaves at harvest & 8.25 & $12.38^{* *}$ & 1.31 & 9.08 \\
\hline Mean leaf length $(\mathrm{cm})$ & 7.38 & $750.94^{\star *}$ & 39.25 & 4.16 \\
\hline Mean leaf breadth $(\mathrm{cm})$ & 16.43 & $142.46^{\star *}$ & 15.75 & 6.64 \\
\hline Mean bunch weight $(\mathrm{kg})$ & 1.79 & $19.37^{* *}$ & 5.81 & 27.43 \\
\hline Mean number of hand per bunch & 0.07 & $3.03^{* *}$ & 0.56 & 12.47 \\
\hline Mean hand weight $(\mathrm{g})$ & 34511.16 & $112734.61^{*}$ & 6058.68 & 16.44 \\
\hline Mean number of finger per hand & 21.49 & $3855.54^{\star \star}$ & 144.44 & 21.24 \\
\hline Mean finger weight $(\mathrm{g})$ & 218.52 & $1539.08^{* *}$ & 287.49 & 19.80 \\
\hline Mean peel weight (g) & 43.50 & $248.66^{\star *}$ & 43.05 & 25.56 \\
\hline Mean pulp weight (g) & 71.17 & $622.68^{* *}$ & 144.23 & 20.03 \\
\hline Mean finger length $(\mathrm{cm})$ & 1.87 & $15.73^{* *}$ & 1.57 & 8.74 \\
\hline Mean finger diameter $(\mathrm{cm})$ & 0.09 & $0.32^{* *}$ & 0.08 & 9.17 \\
\hline Finger yield per hectare (ton) & 22.60 & $25.14^{*}$ & 8.91 & 22.84 \\
\hline Total soluble sugar & 0.35 & $8.58^{* *}$ & 1.24 & 6.90 \\
\hline Titrable acid & 0.26 & $0.93^{\star *}$ & 0.21 & 14.78 \\
\hline Total soluble sugar to titrable acid ratio & 6.78 & $14.92^{* *}$ & 3.86 & 15.01 \\
\hline
\end{tabular}
Rep $=$ replication, Geno $=$ genotypes, CV $(\%)=$ coefficient of variation in percent 
Table 2: Mean performance of 15 desert banana genotypes for morphological traits (growth habits)

\begin{tabular}{llllllll}
\hline Clone & PSH & GS & TIBSLE & NSUTS & NLH & LL & LB \\
\hline Kamara Mesenge & 3.48 & 75.6 & 9.4 & 10 & 13.6 & 153.2 & 64.4 \\
Pissang Raja & 3.63 & 77.6 & 7.6 & 7.4 & 13.4 & 161.0 & 61.6 \\
Lactone & 2.26 & 75.0 & 6.2 & 5.4 & 12.4 & 156.0 & 60.8 \\
Butuza & 1.16 & 75.6 & 7.4 & 5.8 & 12.0 & 144.6 & 53.0 \\
Grande Naine & 1.31 & 65.2 & 6.0 & 4.8 & 11.4 & 145.8 & 52.8 \\
Poyo & 1.26 & 63.8 & 9.4 & 5.4 & 11.4 & 139.2 & 59.0 \\
Dwarf Cavendish & 1.54 & 58.2 & 6.4 & 7.0 & 13.0 & 152.2 & 51.4 \\
Giant Cavendish & 1.67 & 65.2 & 8.8 & 7.0 & 14.6 & 162.8 & 66.0 \\
Williams 2 & 1.15 & 67.6 & 8.8 & 4.2 & 9.0 & 141.4 & 67.0 \\
Robusta & 1.44 & 65.2 & 8.6 & 6.4 & 12.8 & 163.4 & 62.4 \\
Williams 1 & 1.95 & 73.0 & 10.0 & 7.0 & 13.8 & 157.2 & 57.4 \\
Mean of introduced & 1.90 & 69.27 & 8.05 & 6.40 & 12.49 & 152.44 & 59.62 \\
Hararghe clone 1 & 1.54 & 66.2 & 8.8 & 8.0 & 12.2 & 148.0 & 52.6 \\
Hararghe clone 2 & 3.28 & 63.4 & 7.6 & 7.8 & 15.6 & 168.4 & 66.8 \\
Hararghe clone 3 & 1.55 & 54.0 & 8.4 & 7.0 & 12.8 & 145.2 & 60.2 \\
Hararghe clone 4 & 1.25 & 64.0 & 9.4 & 4.4 & 11.2 & 119.4 & 61.6 \\
Mean of local collection & 1.91 & 61.90 & 8.55 & 6.80 & 12.95 & 145.25 & 60.30 \\
SD & 0.86 & 6.80 & 1.26 & 1.55 & 1.57 & 12.26 & 5.34 \\
Grand Mean & 1.90 & 67.31 & 8.19 & 6.51 & 12.61 & 150.52 & 59.80 \\
\hline
\end{tabular}

PSH $(\mathrm{m})=$ mean pseudostem height $(\mathrm{m}), \mathrm{GS}(\mathrm{cm})=$ pseudostem girth, TIBSLE = time interval between successive leaf emergence,

NSUTS $=$ number of suckers until time of shooting, NLH = number of leaves at harvest, LL $(\mathrm{cm})=$ leaf length $(\mathrm{cm}), \mathrm{LB}(\mathrm{cm})=$ leaf breadth $(\mathrm{cm})$

Table 3: Mean performance of 15 desert banana genotypes for fruit yield and yield components

\begin{tabular}{lllllllll}
\hline Genotype & MBW & MNH & MWH & MNF/H & MFW & MFL & MFD & MFY \\
\hline Kamara Mesenge & 10.00 & 6 & 1088.80 & 23 & 48.08 & 10.55 & 2.60 & 10.75 \\
Pissang Raja & 8.08 & 6 & 1190.92 & 13 & 100.41 & 14.87 & 3.39 & 12.34 \\
Lactone & 5.62 & 5 & 869.10 & 10 & 82.78 & 13.68 & 3.41 & 6.68 \\
Butuza & 6.00 & 5 & 877.00 & 11 & 83.60 & 15.81 & 2.89 & 7.59 \\
Grande Naine & 10.12 & 6 & 984.40 & 10 & 100.52 & 15.50 & 3.02 & 9.71 \\
Poyo & 6.94 & 5 & 835.23 & 11 & 77.34 & 14.54 & 2.83 & 6.66 \\
Dwarf Cavendish & 7.92 & 6 & 911.60 & 10 & 84.11 & 15.00 & 2.92 & 8.47 \\
Giant Cavendish & 6.66 & 6 & 1052.28 & 15 & 71.07 & 13.97 & 2.76 & 10.44 \\
Williams 2 & 11.02 & 6 & 944.33 & 13 & 79.66 & 15.49 & 2.98 & 9.60 \\
Robusta & 9.28 & 5 & 1011.26 & 9 & 121.45 & 16.42 & 3.25 & 8.37 \\
Williams 1 & 8.18 & 7 & 843.60 & 11 & 80.65 & 14.39 & 3.02 & 9.72 \\
Mean of introduced & 8.17 & 5.73 & 964.41 & 12 & 84.52 & 14.57 & 3.01 & 9.12 \\
Hararghe clone 1 & 12.22 & 8 & 976.00 & 16 & 68.25 & 11.73 & 3.11 & 13.98 \\
Hararghe clone 2 & 8.82 & 6 & 594.00 & 17 & 87.55 & 11.62 & 3.49 & 14.29 \\
Hararghe clone 3 & 9.68 & 5 & 1060.00 & 13 & 107.68 & 16.41 & 3.26 & 11.20 \\
Hararghe clone 4 & 11.20 & 6 & 724.00 & 13 & 90.93 & 15.15 & 3.12 & 11.35 \\
Mean of local collection & 10.48 & 6.25 & 838.5 & 15 & 88.60 & 13.73 & 3.25 & 12.70 \\
SD & 1.97 & 0.83 & 150.16 & 3.70 & 17.54 & 1.77 & 0.25 & 2.34 \\
Grand Mean & 8.78 & 5.87 & 930.83 & 13.00 & 85.61 & 14.34 & 3.07 & 10.08 \\
\hline
\end{tabular}

$\mathrm{MBW}(\mathrm{kg})=$ mean bunch weight, $\mathrm{MNH}=$ mean number of hand per bunch, $\mathrm{MWH}(\mathrm{g})=$ mean hand weight, $\mathrm{MNF}=$ mean number of finger per hand, $\operatorname{MFW}(\mathrm{g})=$ mean finger weight, $\mathrm{MFL}(\mathrm{cm})=$ mean finger length, $\operatorname{MFD}(\mathrm{cm})=$ mean finger diameter, $\mathrm{MFY}(\mathrm{t})=$ finger yield per hectare.

Table 4: Mean performance of 15 desert banana genotypes for fruit physicochemical traits

\begin{tabular}{llllll}
\hline Genotype & MPelW & MPulpW & TSS & TA & TSS/TA \\
\hline Kamara Mesenge & 10.24 & 37.84 & 17.88 & 2.88 & 7.10 \\
Pissang Raja & 33.51 & 66.90 & 15.12 & 2.60 & 6.04 \\
Lactone & 24.52 & 58.26 & 16.00 & 1.93 & 8.34 \\
Butuza & 20.73 & 62.87 & 18.00 & 1.60 & 11.25 \\
Grande Naine & 32.30 & 68.22 & 15.48 & 1.76 & 8.81 \\
Poyo & 25.13 & 52.21 & 14.40 & 1.60 & 9.00 \\
Dwarf Cavendish & 23.84 & 60.28 & 15.84 & 1.80 & 8.80 \\
Giant Cavendish & 24.37 & 46.70 & 15.60 & 1.65 & 9.90 \\
Williams 2 & 24.55 & 55.12 & 17.40 & 1.76 & 10.95 \\
Robusta & 38.52 & 82.94 & 17.16 & 1.88 & 10.18 \\
Williams 1 & 22.46 & 58.19 & 16.56 & 1.28 & 13.02 \\
Mean of introduced & 25.47 & 59.05 & 16.31 & 1.89 & 9.40 \\
Hararghe clone 1 & 16.92 & 51.33 & 18.36 & 2.32 & 7.95 \\
Hararghe clone 2 & 24.96 & 62.59 & 15.00 & 1.47 & 10.51 \\
Hararghe clone 3 & 31.78 & 75.91 & 14.70 & 1.65 & 9.14 \\
Hararghe clone 4 & 31.22 & 59.71 & 14.90 & 1.61 & 9.61 \\
Mean of local collection & 26.22 & 62.39 & 15.74 & 1.76 & 9.30 \\
SD & 7.05 & 11.16 & 1.31 & 0.43 & 1.73 \\
Grand Mean & 25.67 & 59.94 & 16.16 & 1.85 & 9.37 \\
\hline Mpl(g)= mean peel weight, Mpulp(g)=mean pulp weight, TSS(\%)=total soluble sugar and \\
\multicolumn{1}{r}{ TA(\%)=titrable acid, TSS/TA= total soluble sugar to titrable acid ratio }
\end{tabular}


Table 5: Genotypic and phenotypic coefficient of variances, heritability and genetic advance in 15 desert banana genotypes for 20 morpho-physicochemical traits

\begin{tabular}{|c|c|c|c|c|c|c|}
\hline Trait & $\sigma^{2} g$ & $\sigma^{2} p$ & GCV & PCV & $\mathrm{H}_{2}$ & GA (10\%) \\
\hline Mean pseudostem height (m) & 0.78 & 0.80 & 46.75 & 47.22 & 98.00 & 81.45 \\
\hline Girth of pseudostem (cm) & 39.34 & 74.16 & 9.32 & 12.79 & 53.04 & 11.94 \\
\hline Time interval between successive leaf emergence & 1.38 & 2.46 & 14.32 & 19.14 & 56.01 & 18.87 \\
\hline Number of suckers until time of shooting & 1.75 & 4.98 & 20.31 & 34.29 & 35.09 & 21.18 \\
\hline Mean number of leaves at harvest & 2.21 & 3.53 & 11.80 & 14.89 & 62.82 & 16.46 \\
\hline Mean leaf length $(\mathrm{cm})$ & 142.34 & 181.59 & 7.93 & 8.95 & 78.38 & 12.35 \\
\hline Mean leaf breadth (cm) & 25.34 & 41.09 & 8.42 & 10.72 & 61.67 & 11.64 \\
\hline Mean bunch weight (kg) & 2.71 & 8.52 & 18.75 & 33.23 & 31.84 & 18.62 \\
\hline Mean number of hand per bunch & 0.49 & 1.05 & 11.71 & 17.11 & 46.88 & 14.11 \\
\hline Mean hand weight $(\mathrm{g})$ & 10429.59 & 71016.26 & 10.97 & 28.63 & 14.69 & 7.40 \\
\hline Mean number of finger per hand & 742.22 & 886.66 & 48.16 & 52.63 & 83.71 & 77.54 \\
\hline Mean finger weight $(\mathrm{g})$ & 250.32 & 537.81 & 18.48 & 27.09 & 46.54 & 22.19 \\
\hline Mean peel weight $(\mathrm{g})$ & 41.12 & 84.17 & 24.98 & 35.74 & 48.85 & 30.73 \\
\hline Mean pulp weight $(\mathrm{g})$ & 95.69 & 239.92 & 16.32 & 25.84 & 39.88 & 18.14 \\
\hline Mean finger length (cm) & 2.83 & 4.40 & 11.74 & 14.63 & 64.33 & 16.57 \\
\hline Mean finger diameter (cm) & 0.05 & 0.13 & 7.20 & 11.66 & 38.11 & 7.82 \\
\hline Finger yield per hectare (ton) & 2.85 & 13.75 & 16.78 & 36.88 & 20.70 & 13.43 \\
\hline Total soluble sugar & 1.47 & 2.71 & 7.50 & 10.19 & 54.12 & 9.71 \\
\hline Titrable acid & 0.14 & 0.36 & 20.41 & 32.27 & 39.99 & 22.71 \\
\hline Total soluble sugar to titrable acid ratio & 2.21 & 6.07 & 15.87 & 26.29 & 36.42 & 16.85 \\
\hline
\end{tabular}

Min=minimum, Max=maximum, SD=standard deviation, $\sigma^{2} \mathrm{~g}=$ genotypic variance, $\sigma^{2} \mathrm{p}=$ =phenotypic variance, GCV=genotypic coefficient of variance, $\mathrm{H}_{2}=$ heritability, $\mathrm{PCV}=$ phenotypic coefficient of variance, $\mathrm{GA}=$ expected genetic advance at $10 \%$.

High heritability in broad sense was recorded for six traits in the range between 61.67 (mean leaf breadth) to $98 \%$ (mean pseudostem height). Low heritability was recorded for mean hand weight (14.69\%) and finger yield per hectare $(20.7 \%)$, while moderate heritability (31.84$56.01 \%$ ) was registered for the remaining 12 out of 20 traits. The highest genetic advance as per cent mean $(81.45 \%)$ was recorded for mean pseudostem height and other five traits (mean number of finger per hand, mean peel weight, titrable acid, mean finger weight and number of suckers until time of shooting). Three traits viz., mean hand weight $(7.4 \%)$, mean finger diameter $(7.82 \%)$ and total soluble solid $(9.71 \%)$ registered low genetic advance as per cent mean. The remaining 11 out of 20 traits exhibited moderate (11.64 to $18.87 \%$ ) genetic advance as percent mean at $10 \%$ selection intensity (Table 5 ).

\section{Phenotypic and Genotypic Correlations}

All possible pairs of phenotypic and genotypic correlation coefficients were computed for 20 morphophysicochemical traits and are presented in Table 6 . Fruit yield per hectare had significant and positive genotypic correlations with all growth traits viz., mean pseudostem height, girth of pseudostem, time interval between successive leaf emergence, number of suckers until time of shooting, mean number of leaves at harvest and mean leaf breadth. However, the correlation between fruit yield per hectare and leaf length was positive but nonsignificant. Pseudostem height and time interval between successive leaf emergence with mean bunch weight and number of suckers until time of shooting and leaf length with mean hand weight showed positive and significant genotypic association. Mean number of hand per bunch with time interval between successive leaf emergence and number of suckers until time of shooting and mean number of finger per hand with girth of pseudostem and leaf length had positive and significant genotypic correlation. Pseudostem height with mean hand weight and mean number of finger per hand, girth of pseudostem with mean bunch weight and mean finger weight showed negative and significant genotypic correlations,. Similarly, time interval between successive leaf emergence and number of suckers until time of shooting with mean finger weight, number of leaf at harvest and leaf length with mean bunch weight had negative and significant genotypic correlations.

Fruit yield per hectare showed significant and positive genotypic correlations with mean bunch weight, mean number of hand per bunch, mean finger diameter, mean number of finger per hand and total soluble solid. However, fruit yield per hectare has negative and significant genotypic associations with mean finger length and total soluble solid to titrable acid ratio. Total soluble solid and total soluble solid to titrable acid ratio showed significant and positive genotypic correlations. Total soluble solid and total soluble solid to titrable acid ratio also exhibited positive and significant genotypic associations with mean bunch weight and mean hand weight while these traits had negative and significant genotypic correlations with mean peel and pulp weight as well as with finger length.

Fruit yield per hectare showed positive and significant phenotypic associations with mean bunch weight, number of hand per bunch, hand weight and finger diameter. Titrable acid with mean hand weight exhibited positive and significant phenotypic associations. It has been observed significant phenotypic associations in considerable pairs of yield components and fruit physical attributes (Table 6). On the other hand, total soluble solid with mean finger weight, peel weight and finger length and total soluble solid to titrable acid ratio with titrable acid showed negative and significant phenotypic correlations.

Pseudostem height had positive and significant phenotypic correlation with fruit yield per hectare, yield components (bunch weight, hand weight, number of finger per hand, finger diameter) and number of leaves at harvest, but it showed negative and significant correlation with finger length and pseudostem girth. Pseudostem girth exhibited positive and significant phenotypic correlations with number of finger per hand, total soluble sugar and titrable acid, while it showed negative associations with 
Wassu Mohammed et alo,

mean bunch weight, finger weight, pulp weight and finger length. Time interval between successive leaf emergence with mean bunch weight and number of hand per bunch, number of suckers until time of shooting with total soluble solid and titrable acid showed positive and significant phenotypic correlations. Number of suckers until time of shooting with mean finger weight, finger length, peel and pulp weight, and number of leaves at harvest with mean
Sci. Technol. Arts Res. J., Oct-Dec 2014, 3(4): 19-28

finger length exhibited negative and significant phenotypic associations. Leaf length had positive and significant phenotypic correlation with mean number of finger per hand while leaf length exhibited negative associations with mean bunch weight and finger length. In addition, growth traits showed significant phenotypic associations each other in both directions (Table 6).

Table 6: Genotypic and phenotypic correlation coefficients above diagonal and below diagonal, respectively, for 20 morpho-physicochemical traits of 15 desert banana genotypes

\begin{tabular}{|c|c|c|c|c|c|c|c|c|c|c|}
\hline Trait & PSH & GS & TIBSLE & NSUTS & NLH & LL & LB & MBW & MNH & MWH \\
\hline$\overline{\mathrm{PSH}}$ & & $-0.42^{\star \star}$ & 0.05 & $0.23^{*}$ & $0.36^{\star *}$ & 0.05 & $0.21^{*}$ & $0.37^{\star \star}$ & 0.13 & $-0.53^{\star \star}$ \\
\hline GS & $-0.30^{*}$ & & 0.02 & 0.15 & 0.03 & $0.21^{*}$ & 0.08 & $-0.30^{*}$ & 0.14 & 0.19 \\
\hline TIBSLE & 0.04 & 0.08 & & $0.20^{*}$ & -0.01 & $-0.22^{*}$ & $0.38^{* *}$ & $0.32^{*}$ & $0.38^{\star *}$ & -0.01 \\
\hline NSUTS & 0.18 & 0.16 & 0.19 & & $0.72^{\star \star}$ & $0.56^{\star *}$ & 0.08 & 0.06 & $0.26^{*}$ & $0.31^{*}$ \\
\hline NLH & $0.30^{*}$ & 0.05 & 0.00 & $0.49^{*}$ & & $0.74^{\star *}$ & 0.18 & $-0.33^{*}$ & 0.02 & -0.05 \\
\hline LL & 0.04 & 0.11 & -0.18 & $0.29^{*}$ & $0.52^{*}$ & & $0.24^{*}$ & $-0.37^{* *}$ & -0.06 & $0.21^{*}$ \\
\hline LB & 0.17 & 0.02 & $0.26^{*}$ & 0.04 & 0.01 & $0.26^{*}$ & & 0.04 & -0.13 & -0.03 \\
\hline MBW & $0.25^{*}$ & $-0.24^{*}$ & $0.24^{*}$ & 0.05 & -0.02 & $-0.20^{*}$ & -0.03 & & $0.61^{* *}$ & 0.06 \\
\hline MNH & 0.09 & 0.02 & $0.21^{*}$ & 0.06 & 0.12 & -0.02 & -0.11 & $0.51^{*}$ & & 0.12 \\
\hline MWH & $0.30^{*}$ & -0.06 & -0.06 & -0.07 & -0.06 & 0.04 & -0.08 & 0.17 & 0.16 & \\
\hline MNFH & $0.77^{\star *}$ & $0.29^{*}$ & -0.10 & -0.05 & -0.02 & $0.29^{*}$ & 0.04 & -0.19 & 0.10 & $0.24^{*}$ \\
\hline MFW & 0.08 & $-0.21^{*}$ & -0.11 & $-0.30^{*}$ & -0.10 & 0.06 & 0.06 & 0.09 & $-0.22^{*}$ & $0.22^{*}$ \\
\hline MPelW & 0.01 & -0.18 & -0.11 & $-0.36^{*}$ & -0.10 & 0.03 & 0.15 & 0.03 & -0.19 & 0.13 \\
\hline MPulpW & 0.11 & $-0.21^{*}$ & -0.10 & $-0.23^{*}$ & -0.09 & 0.07 & -0.01 & 0.11 & $-0.22^{*}$ & $0.26^{*}$ \\
\hline MFL & $-0.30^{*}$ & $-0.20^{*}$ & -0.08 & $-0.48^{*}$ & $-0.34^{*}$ & $-0.24^{*}$ & -0.09 & -0.03 & $-0.23^{*}$ & $0.25^{\star}$ \\
\hline MFD & $0.37^{*}$ & 0.01 & -0.10 & -0.12 & 0.07 & 0.17 & 0.17 & 0.09 & -0.14 & 0.01 \\
\hline MFY & $0.37^{\star}$ & -0.10 & 0.04 & 0.09 & 0.10 & -0.07 & -0.01 & $0.36^{*}$ & $0.45^{\star}$ & $0.37^{*}$ \\
\hline TSS & -0.19 & $0.33^{*}$ & 0.18 & $0.20^{*}$ & -0.11 & 0.12 & -0.09 & 0.16 & 0.14 & 0.01 \\
\hline TA & -0.14 & $0.27^{*}$ & 0.05 & $0.25^{*}$ & -0.09 & 0.07 & 0.14 & 0.08 & 0.01 & $0.23^{*}$ \\
\hline TSST & 0.05 & -0.08 & 0.10 & -0.13 & 0.14 & 0.01 & -0.14 & -0.01 & 0.04 & -0.16 \\
\hline
\end{tabular}

$\mathrm{PSH}(\mathrm{m})=$ mean pseudostem height $(\mathrm{m}), \mathrm{GS}(\mathrm{cm})=$ pseudostem girth, TIBSLE $=$ time interval between successive leaf emergence

NSUTS = number of suckers until time of shooting, $\mathrm{NLH}=$ number of leaves at harvest, $\mathrm{LL}(\mathrm{cm})=$ leaf length $(\mathrm{cm})$,

$\mathrm{LB}(\mathrm{cm})=$ leaf breadth $(\mathrm{cm}), \mathrm{MBW}(\mathrm{kg})=$ mean bunch weight, $\mathrm{MNH}=$ mean number of hand per bunch, $\mathrm{MWH}(\mathrm{g})=\mathrm{mean}$ hand weight

\begin{tabular}{|c|c|c|c|c|c|c|c|c|c|c|}
\hline Trait & MNFH & MFW & MPelW & MPulpW & MFL & MFD & MFY & TSS & TA & TSS/TA \\
\hline $\mathrm{PSH}$ & $-0.83^{\star *}$ & 0.08 & 0.01 & 0.13 & $-0.37^{*}$ & $0.52^{*}$ & $0.67^{\star *}$ & $-0.24^{\star}$ & -0.19 & 0.06 \\
\hline GS & $0.43^{*}$ & $-0.34^{*}$ & $-0.33^{*}$ & $-0.32^{*}$ & $-0.28^{*}$ & -0.06 & -0.14 & $0.44^{*}$ & $0.43^{*}$ & -0.12 \\
\hline TIBSLE & -0.03 & $-0.30^{*}$ & $-0.22^{*}$ & $-0.34^{*}$ & -0.17 & $-0.35^{*}$ & $0.25^{*}$ & 0.14 & -0.02 & $0.28^{*}$ \\
\hline NSUTS & -0.08 & $-0.40^{*}$ & $-0.51^{*}$ & $-0.32^{*}$ & $-0.68^{* *}$ & -0.12 & $0.41^{*}$ & $0.27^{*}$ & $0.56^{*}$ & $-0.35^{*}$ \\
\hline $\mathrm{NLH}$ & -0.05 & -0.08 & -0.12 & -0.04 & $-0.45^{\star}$ & 0.18 & $0.30^{*}$ & -0.18 & 0.04 & -0.04 \\
\hline LL & $0.36^{*}$ & 0.06 & -0.02 & 0.10 & $-0.29^{*}$ & $0.27^{*}$ & 0.02 & 0.10 & 0.15 & -0.02 \\
\hline LB & 0.05 & -0.07 & 0.08 & -0.16 & $-0.21^{*}$ & 0.14 & $0.21^{*}$ & -0.18 & 0.07 & 0.04 \\
\hline MBW & $-0.41^{*}$ & 0.01 & 0.01 & 0.01 & -0.15 & 0.02 & $0.66^{\star *}$ & $0.23^{*}$ & $0.25^{\star}$ & -0.12 \\
\hline MNH & -0.03 & $-0.34^{*}$ & $-0.31^{*}$ & $-0.34^{*}$ & $-0.34^{*}$ & -0.15 & $0.67^{* *}$ & $0.34^{*}$ & 0.19 & 0.05 \\
\hline $\mathrm{MWH}$ & $0.43^{*}$ & 0.04 & 0.07 & 0.03 & 0.15 & $-0.23^{*}$ & 0.02 & $0.22^{*}$ & $0.65^{\star *}$ & $-0.53^{*}$ \\
\hline MNFH & & -0.04 & 0.05 & -0.09 & $0.25^{\star}$ & $-0.40^{*}$ & $0.52^{*}$ & 0.19 & 0.07 & 0.13 \\
\hline MFW & -0.04 & & $0.94^{* *}$ & $0.98^{\star *}$ & $0.75^{\star *}$ & $0.63^{*}$ & 0.06 & $-0.38^{*}$ & $-0.32^{*}$ & 0.11 \\
\hline MPelW & 0.04 & $0.90^{\star *}$ & & $0.85^{\star \star}$ & $0.74^{\star *}$ & $0.56^{*}$ & 0.04 & $-0.54^{*}$ & $-0.31^{*}$ & 0.04 \\
\hline MPulpW & -0.09 & $0.97^{\star *}$ & $0.76^{\star \star}$ & & $0.71^{\star *}$ & $0.64^{*}$ & 0.07 & $-0.26^{*}$ & $-0.31^{*}$ & 0.15 \\
\hline MFL & 0.17 & $0.76^{\star *}$ & $0.73^{\star *}$ & $0.71^{\star \star}$ & & 0.13 & $-0.41^{*}$ & $-0.26^{*}$ & $-0.46^{*}$ & $0.30^{*}$ \\
\hline MFD & $-0.27^{*}$ & $0.72^{\star *}$ & $0.64^{*}$ & $0.71^{\star *}$ & $0.34^{*}$ & & $0.28^{\star}$ & $-0.33^{*}$ & -0.12 & -0.07 \\
\hline MFY & 0.16 & 0.13 & 0.08 & 0.15 & -0.07 & $0.21^{*}$ & & -0.03 & $0.26^{*}$ & $-0.23^{*}$ \\
\hline TSS & 0.14 & $-0.21^{*}$ & $-0.35^{\star}$ & -0.11 & $-0.22^{*}$ & -0.17 & -0.11 & & $0.37^{*}$ & 0.12 \\
\hline TA & 0.05 & -0.04 & -0.01 & -0.06 & -0.17 & 0.07 & 0.08 & 0.19 & & $-0.83^{* *}$ \\
\hline TSST & 0.06 & -0.08 & -0.12 & -0.05 & 0.09 & -0.15 & -0.09 & 0.08 & $-0.83^{\star *}$ & \\
\hline
\end{tabular}

$\mathrm{MNFH}=$ mean number of finger per hand, MFW $(\mathrm{g})=$ mean finger weight, $\mathrm{Mpel}(\mathrm{g})=$ mean peel weight, $\mathrm{Mpulp}(\mathrm{g})=$ mean pulp weight, $\mathrm{MFL}(\mathrm{cm})=$ mean finger length, $\operatorname{MFD}(\mathrm{cm})=$ mean finger diameter, $\mathrm{MFY}(\mathrm{t})=$ finger yield per hectare,

$\mathrm{TSS}(\%)=$ total soluble sugar and $\mathrm{TA}(\%)=$ titrable acid, TSS/TA $=$ total soluble sugar to titrable acid ratio.

\section{Genetic Distance of Desert Banana Genotypes}

Genetic distances among desert banana genotypes were estimated using Euclidean distance and are presented in Table 7. Euclidean distance ranged from 2.36 to 7.6 with mean and standard deviation of 4.87 and 1.04 , respectively. The most distant genotypes were Kamaramesenge and Robusta (7.6) closely followed by Kamaramesenge and Hararghe clone 3 (7.58) and Kamaramesenge and Hararghe clone 4 (7.47). The lowest distance was computed between Grande Naine and
Dwarf Cavendish (2.36) Followed by Giant Cavendish and Williams 1 (3.06) and Butuza and Dwarf Cavendish (3.1). On the basis of mean Euclidean distance, Kamaramesenge (6.35) followed by Hararghe clone 2 (5.53), Hararghe clone 1 (5.35) and Pissang Raja (5.31) were the most distant genotypes to others. Dwarf Cavendish (4.16) followed by Grande Naine (4.35) were the closest genotypes to others. The local banana collections were relatively the more distant to others except Hararghe clone 3 (4.77). 
Table 7: Euclidean distance of 15 desert banana genotypes measured from 20 morpho-physicochemical traits and mean Euclidean distance obtained by averaging each genotype distance to other 14

\begin{tabular}{lccccccccccccccc}
\hline & C2 & C3 & C4 & C5 & C6 & C7 & C8 & C9 & C10 & C11 & C12 & C13 & C14 & C15 & Mean ED \\
\hline C1 & 5.72 & 6.00 & 6.49 & 7.22 & 6.33 & 6.21 & 5.02 & 6.44 & 7.60 & 5.81 & 4.80 & 6.28 & 7.58 & 7.47 & 6.35 \\
C2 & & 4.17 & 5.76 & 4.75 & 5.73 & 4.94 & 4.76 & 5.90 & 4.63 & 5.24 & 5.93 & 5.14 & 5.27 & 6.35 & 5.31 \\
C3 & & & 3.44 & 3.84 & 3.76 & 3.47 & 4.14 & 4.77 & 4.40 & 4.72 & 5.65 & 4.53 & 4.72 & 5.42 & 4.50 \\
C4 & & & & 3.45 & 3.49 & 3.10 & 4.29 & 3.90 & 4.35 & 3.68 & 5.17 & 6.17 & 4.90 & 4.98 & 4.51 \\
C5 & & & & & 3.70 & 2.36 & 4.56 & 3.86 & 3.69 & 4.53 & 5.15 & 5.91 & 3.64 & 4.17 & 4.35 \\
C6 & & & & & 3.23 & 3.59 & 3.68 & 4.70 & 4.19 & 5.57 & 5.78 & 4.06 & 3.78 & 4.40 \\
C7 & & & & & & 3.60 & 4.49 & 4.16 & 4.05 & 4.69 & 5.21 & 3.75 & 4.89 & 4.16 \\
C8 & & & & & & & 4.30 & 4.58 & 3.06 & 5.27 & 4.72 & 4.92 & 5.54 & 4.45 \\
C9 & & & & & & & & 4.52 & 4.15 & 5.12 & 6.50 & 4.97 & 3.94 & 4.75 \\
C10 & & & & & & & & & 4.56 & 6.38 & 5.63 & 3.69 & 5.48 & 4.88 \\
C11 & & & & & & & & & & 4.87 & 5.03 & 5.25 & 5.21 & 4.60 \\
C12 & & & & & & & & & & & 5.73 & 5.51 & 5.07 & 5.35 \\
C13 & & & & & & & & & & & & 4.90 & 5.93 & 5.53 \\
C14 & & & & & & & & & & & & & 3.63 & 4.77 \\
C15 & & & & & & & & & & & & & & 5.13 \\
\hline
\end{tabular}

C1 = Kamara Mesenge, C2= Pissang Raja, C3= Lactone, C4= Butuza, C5 = Grande Naine, C6= Poyo, C7= Dwarf Cavendish, C8= Giant Cavendish, $\mathrm{C} 9=$ Williams 2, C10= Robusta, $\mathrm{C} 11=$ Williams $1, \mathrm{C} 12=$ Hararghe clone $1, \mathrm{C} 13=$ Hararghe clone 2, C14= Hararghe clone 3 and $\mathrm{C} 15=$ Hararghe clone 4, Mean ED = mean Euclidean distance.

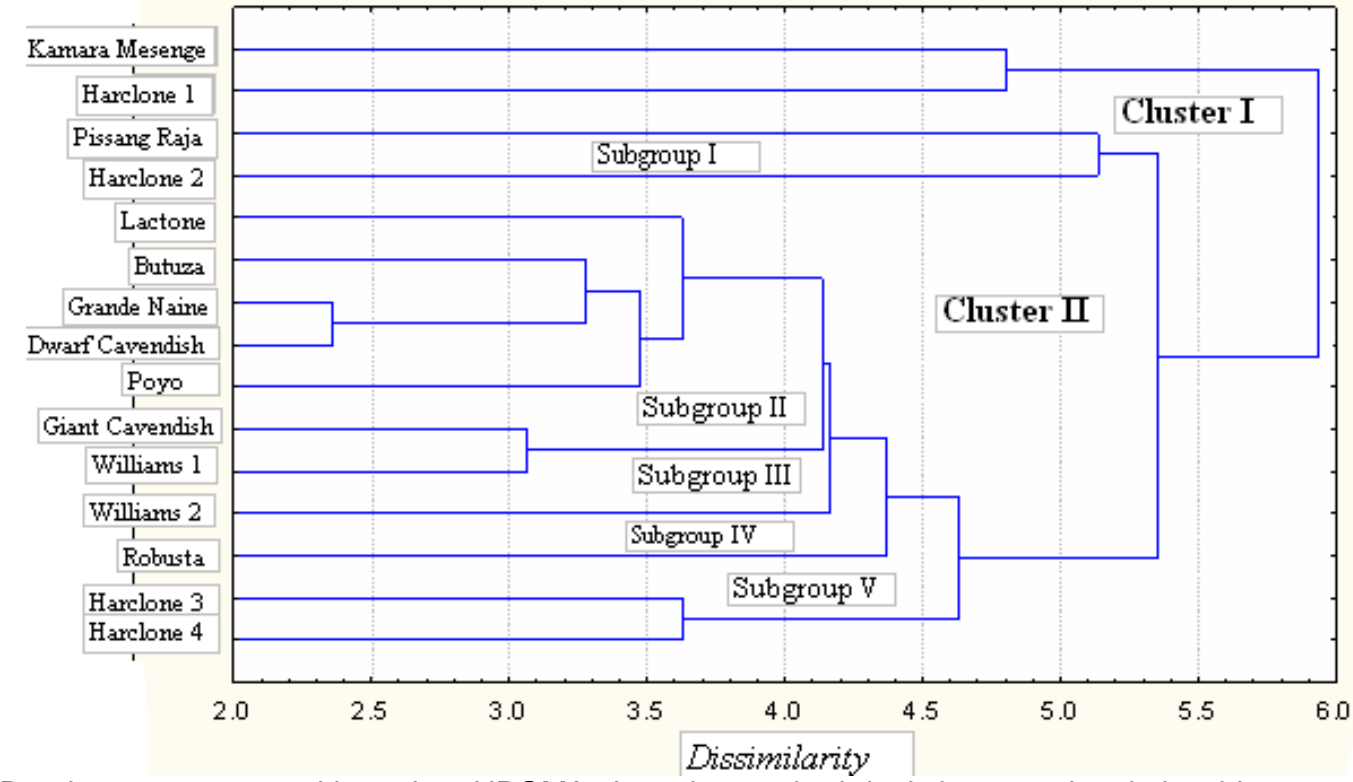

Figure 1: Dendrogram generated based on UPGMA clustering method depicting genetic relationships among 15 desert banana genotypes based on 20 morpho-physicochemical traits

The dendrograms from unweighted Pair-group Method with Arithmetic means (UPGMA) are presented in Figure 1. The genotypes were grouped in two clusters of which the first cluster comprised of Kamaramesenge and Hararghe clone 1, while the rest of the 13 genotypes were formed the second cluster. The second cluster was subgrouped in to five, which the first subgroup consisted of Pissang Raja and Hararghe clone 2. The second subgroup consisted of relatively large number of genotypes (Lactane, Butuza, Grande Naine, Dwarf Cavendish, Poyo, Giant Cavendish and Williams 1), which most of the members are the Cavendish type of desert banana. Williams 2 and Robusta stands separately (solitary) which formed sub-group 3 and 4, respectively, while the two Hararghe collections, Hararghe clone 3 and 4 were formed the fifth sub-group.
The first cluster that consisted of Kamaramesenge and Hararghe clone 1 is characterized by highest time interval between successive leaf emergence, number of suckers until time of shooting, mean bunch weight, number of hand per bunch, number of finger per hand, total soluble solid and titrable acid. It is also characterized by having lowest mean values for finger weight, peel and pulp weight, as well as the second and third lowest total soluble solid to titrable acid ratio. The second cluster consisted of clones which had a wide range of mean values for all traits, but it can be characterized with higher total soluble solid to titrable acid ratio except one clone. Clones which registered highest and lowest fruit yield are also included in this cluster.

The first subgroup (Pissang Raja and Hararghe clone 2 ) in the second cluster is characterized by having the highest mean values for pseudostem height, number of 
Wassu Mohammed et al.,

leaves at harvest, leaf length, leaf breadth, number of hand per bunch and finger diameter. The two genotypes also recorded the first and third highest mean fruit yield per hectare in the experiment. The second sub-group which consists of the Cavendish desert banana type registered average mean values for most of the traits. The sub-group 3 (Williams 2) is characterized by recording the lowest mean values for pseudostem height, number of suckers until time of shooting, number of leaves at harvest, leaf length but having the largest leaf and total soluble solid to titrable acid ratio. Robusta stands separately (solitary) and formed sub-group 4 and characterized by having longest leaf length, highest mean values for finger weight, peel weight, pulp weight and finger length. The fifth sub-group (Hararghe clone 3 and 4 ) is characterized by having average mean values for most of the traits but recorded the highest mean values for mean finger weight, finger length and the fourth and fifth high yielding clones in the experiment. Though the Hararghe collections were grouped in both clusters, the four Hararghe clones generally characterized by producing high fruit yield per hectare, which ranked the first, second, fourth and fifth highest yield in the experiment.

\section{DISCUSSION}

The analysis of variance revealed significant differences among the clones for all the traits. In addition, estimates of phenotypic and genotypic coefficients of variation, heritability and genetic advance as per cent of mean indicating the presence of variation and genotypes were diverse materials. The presence of variation is critical in banana to improve the crop. Because genetic variability does not arise through crossing since the crop is sterile and parthenocarpic and propagated vegetatively. Genetic variability in banana is created mainly through hybridizations of diploid bananas (Vuylsteke and Swennen, 1993), somaclonal variation (Hwang and Tang, 1996) and induced mutation (Silayoi et al., 1995). However, the usefulness of these methods is limited (Heslop-Harrison and Schwarzacher, 2007), because these methods are time consuming and require high capital. Therefore, selection of cultivars or clones in existing natural variability is relatively low cost and time for the improvement of crop. The adaptability differences of the Cavandishes group (Dwarf Cavandishes, Giant Cavandishes and Poyo) and Ducasse hybrid (Pissang) in the country has been reported. Pissang gave higher yield than Giant Cavandishes ans Poyo relatively under cooler conditions, but its fruit are small and they lack banana flavor (EARO, 1999). Similarly, in this study, Pissang Raja gave higher yield than Giant Cavandishes and Poyo. But Pissang Raja registered the lowest total soluble solid to titrable acid indicating it was not as sweet to be used as desert banana.

The overall mean values of four eastern Ethiopia banana clones were higher than the introduced desert banana genotypes for 13 out of 20 traits. More importantly, these clones produced higher fruit yield than introduced genotypes except Pissang Raja which exceeded the two local clones for yield. The higher performance of eastern Ethiopia banana clones indicating that the higher chance of improving banana yield and other traits through local materials collection and selection. The presence of variations within East African bananas where Ethiopia is as part of the region was also confirmed from other report (JAICAF, 2010). East African
Sci. Technol. Arts Res. J., Oct-Dec 2014, 3(4): 19-28

highland bananas are forming a distinct subgroup of bananas which their ancestors have arrived in African continent as long as 4,000 years ago (Neuman, and Hildebrand, 2009; Lejju, et al., 2006). It is easy to identify the group from other groups with great variability within the group caused by mutations and high yielding. However, it is largely a subsistence crop, important only for food security without significant export markets (Karamura, 1998).

As proposed by Siva Subramanian and Menon (1973), the computed phenotypic coefficient of variation (PCV) was considered as high which ranged from 25.84 to 52.63 $\%$ for 11 out of 20 traits and moderate for eight that ranged from 10.19 to $19.14 \%$, while low only for leaf length $(8.95 \%)$. Genotypic coefficient of variation (GCV) ranged from 20.31 to $48.16 \%$ for pseudostem height, number of suckers until time of shooting, number of finger per hand, peel weight and titrable acid, which can be considered as high. Low genotypic coefficient of variation $(<10 \%)$ was computed for girth of pseudostem, leaf length, leaf breadth, finger diameter and total soluble solid while moderate (10.97 to $18.75 \%$ ) for the rest of the traits. The estimated phenotypic coefficient of variation was relatively greater than the genotypic coefficient of variation in magnitude for all the traits; however, the differences were low. This indicates the presence of substantial variability; lower sensitivity of traits to environmental modifications and the greater scope of genetic improvement through selection for most of the traits. Sree Rangaswamy et al. (1980) also estimated PCV and GCV in the range between 13 and $29 \%$ for bunch weight, fruit weight and stem girth in a range of dessert banana cultivars. Sirisenaa and Senanayake (2000) reported considerable PCV higher than $15 \%$ for bunch weight, average fruit weight and total fruit weight of which total fruit weight had GCV more than $10 \%$. They also reported that estimated phenotypic coefficient was relatively greater than the genotypic coefficient of variation in magnitude for leaf width, leaf length, bunch weight, pseudostem girth, average fruit weight and number of fruit per hand.

As demonstrated by Robinson et al. (1949), heritability can be categorized as low (0-30\%), moderate (30-60\%) and high (60\% and above) and as Johnson et al., (1955) suggested genetic advance as percent mean can be categorized as low (0-10\%), moderate (10-20\%) and high (20\% and above). In the present study, heritability and genetic advance were in the combinations of high and moderate for 16 out of 20 traits which is an indication of more additive gene action (Panse, 1957). This suggested that these traits are amenable for selection. Single plant selection is much effective for a trait that is highly heritable and heritability estimates would be reliable if accompanied by a high estimate genetic advance (Singh and Choudhry, 1985). On the other hand, in the present study, both heritability and the expected genetic gain upon selecting the best $10 \%$ were low or moderate and low for number of finger per hand, finger diameter, fruit yield per hectare and total soluble solid. This indicating the improvement of these traits through selection is difficult or virtually impractical due to the masking effect of environment on the genotypic effect (Singh, 1990). Higher heritability and expected genetic gain were estimated for bunch weight and average fruit weight (Rekha and Prasad, 1993). Large values for broad sense heritability were computed for plant height, bunch weigh, fruit length 
Wassu Mohammed et al.,

and low values for number of fingers (Ortiz, 1995). Low heritability value and expected genetic advance were computed for number of fruits per hand and pseudostem girth while moderate heritability and expected genetic advance were computed for bunch weight, leaf length and average fruit weight (Sirisenaa and Senanayake, 2000).

Significant and positive genotypic association was observed between fruit yield per hectare and growth traits as well as fruit yield components. It was also evident that the higher magnitude of genotypic correlation coefficients over phenotypic correlation coefficients for these traits. The result suggested that indirect selection of growth traits and fruit yield components may be used to increase fruit yield per hectare. This study result is in agreement with Sirisenaa and Senanayake, (2000) who reported that selection in favour of average fruit weight increases the total fruit weight more than the selection for bunch weight. It is also in agreement with Tenkouano et al. (2002), who reported positive and significant genotypic correlations higher than phenotypic correlation between bunch weight with its components except with number of fingers per hand.

The eastern Ethiopian banana collections; i) Hararghe clone 3 and 4 formed separate sub-group in cluster II, ii) Hararghe clone 2 and 1 grouped in other sub-groups of the two clusters, and iii) all local collections except one were the most distant clones from others. This showed that the local clones are genetically distant each other and with introduced genotypes. Moreover, the overall mean values of local clones were greater than the introduced cultivars for most of the traits may encourage breeders to commence collection and selection of local materials to improve yield and other desirable traits of desert banana. This is in conformity with Swennen et al. (1995) who reported that East Africa is considered as secondary centre of diversity for the Musa AAA group. Pillay et al. (2001) also reported the close relationship of East African banana germplasms, but the presence of sufficient RAPD polymorphisms that were collectively useful in distinguishing the cultivars. East African Highland banana (AAA) is by far the most widely distributed in the region and is said to be endemic to this region with no clear analogue elsewhere in the world (Simmonds, 1959; Shepherd, 1957). In this study, the Cavendish group viz., Dwarf Cavendish, Giant Cavendish, Grand Naine, Williams, Lacatane, Gros Michel and Poyo with AAA genome (Cheesman et al., 1933; Stover and Simmonds 1987; Lebot et. al., 1994; EARO, 1999) were grouped in cluster II and majority of the genotypes were grouped in the second sub-group of the cluster. This showed the efficiency of clustering in grouping of genotypes with similar genome in the same cluster. But Pisang Raja and Kamaramasenge with the AAB genome, (Karamura, 2011; Lebot et al., 1994; Swennen and Vuylsteke 1987) were grouped in different clusters. This might be due to the inclusion of different morphotypes since this sub group was further divided by various workers to clones and morphotypes (Lebot et al., 1994).

\section{CONCLUSIONS}

This study showed that the presence of considerable genetic distance among local banana collections and introduced desert banana genotypes. Relatively, the genetic distance of local clones showed clear relationship with their geographical origin; they were the most distant within and between introduced genotypes. In addition, the
Sci. Technol. Arts Res. J., Oct-Dec 2014, 3(4): 19-28

local collections were high performing clones for most of the traits as compared to introduced genotypes. This indicates the need to collect, evaluate and characterize large number of local clones to improve yield and other desirable traits of desert banana. The presence of substantial variability was evident for all traits studied. Furthermore, the highest values computed for heritability and expected genetic advance for most of the traits and positive and significant genotypic correlation higher than phenotypic correlation was evident between fruit yield and growth traits as well as yield components. This suggested that the higher the chance of improving fruit yield per hectare through selection of clones for growth traits and yield components. The generated information showed that collection of local clones as many as possible; evaluation along with introduced improved varieties and selection of best performing clones is appropriate breeding method rather than evaluation of only introduced materials to improve yield and other desirable traits in target region in particular and in the country.

\section{ACKNOWLEDGMENTS}

The authors are grateful to the financial support of Haramaya University for the execution of the research. The authors also thank the staff members of Horticulture Program, School of Plant Sciences of Haramaya University for their technical assistance during the time of conducting the experiment both at the field and laboratory.

\section{REFERENCES}

Allard, R.W. (1960). Principles of Plant Breeding. John Willy and Sons, Inc., New York, USA.

Burton, G.A. and Devane, E.H. (1953). Estimation of heritability in tall festca (Festuca arundinacea) from replicated clonal materials. Agronomy Journal 45:478479 .

Cheesman, E.E., Wardlaw, C.W and Spencer, G..L. (1933). The Cavendish group of banana varieties. Tropical Agriculture 10: 218-221.

EARO (Ethiopian Agriculture Research Organization). (1999). Banana production and utilization in Ethiopia. Ethiopian Agriculture Research Organization, Research report No. 35, Addis Ababa, Ethiopia.

Falconer, D.S. (1990). Introduction to Quantitative Genetics ( $3^{\text {rd }}$ edition). John Wiley and Sons, Inc., New York, USA.

Gawel, N.J., Jarret, R.L. and Whittemore, A.P. (1992). Restriction fragment length polymorphism (RFLP)based phylogenetic analysis of Musa. Theoretical and Applied Genetics 84:286-290.

Heslop-Harrison, J.S. and Schwarzacher, T. (2007). Domestication, genomics and the future for Banana. Annals of Botany 100(5):1073-1084.

Hwang, S.C., and Tang, C.Y. (1996). Somaclonal variation and its use for improving 'Cavendish' (AAA dessert) banana in Taiwan. In: Annual Report of Taiwan Banana Research Institute, 1995. Taiwan Banana Research Institute, Pingtung, Taiwan, 67-75.

JAICAF (Japan Association for International Collaboration of Agriculture and Forestry). (2010). Cooking Banana in Africa. Edited and Published by Japan Association for International Collaboration of Agriculture and Forestry (JAICAF). Akasaka KSA Building 3F, Akasaka 8-10-39, Minatoku, Tokyo, Japan. pp.1-34. 
Wassu Mohammed et al.,

Johnson, H.W., Robinson, H.F. and Comstock, R.E. (1955). Estimates of genetic and environmental variability in soybeans. Agronomy Journal 47:314-318.

Karamura, D.A. (1998). Numerical taxonomic studies of the eastern African highland bananas (Musa AAAEast Africa) in Uganda. PhD Dissertation, the University of Reading, UK. Available online at http://www.Bioversityinternational.org/publications/pdf/ 142.pdf.

Karamura, D. (2011). Case study: Economic significance of banana varieties other than Cavendish in Uganda, Committee on Commodity Problems Inter governmental Group on Bananas and Tropical Fruits Fifth Session, Yaoundé, Cameroon, 3-5 May 2011. CCP:BA/TF 11/CRS. Yaoundé, Cameroon. pp. 6-11.

Khan, A.J., Azam, F. and Ali, A. (2010). Relationship of morphological traits and grain yield in recombinant inbred wheat lines grown under drought conditions. Pakistan Journal of Botany 42(1): 259-267.

Kotal, B.D., Das, A. and Choudhury, B.K. (2010).Genetic variability and association of characters in wheat (Triticum aestivum L.). Asian Journal of Crop Science 2(3):155-160.

Lebot, V., Meilleur, A.B. and Manshardt, R.M. (1994). Genetic diversity in eastern polynesian Eumusa bananas. Pacific Science 48: 16-31.

Lejju, J.B., Robertshaw, P. and Taylor, D. (2006). Africa's earliest bananas. Journal of Archaeological Science 33:102-113.

Levoyageur Weather: Djibouti. (2012). http://en.wikipedia.org/w/index (Retrieved 11 July 2012).

Miller, P.A., Williams, J.C., Robinson, H.F. and Comstock, R.F. 1958). Estimation of genotypic and environmental variances and covariances in upland cotton and their implications in selection. Agronomy Journal 50: 126131.

Neuman, K. and Hildebrand, E. (2009). Early bananas in Africa: The state of the art. Ethnobotany Research and Applications 7:353-362.

Ortiz R., Ferris, R.S.B. and Vuylsteke, D.R. (1995). In: Gowen, S. (ed.). Banana and plantain breeding. Chapman and Hall, London, UK. pp. 110-146.

Ortiz, R. (1995). Musa genetics. In: Gowen, S. (ed.). Bananas and Plantains. Chapman and Hall, London, UK. pp.84-109.

Panse, U.G. (1957). Genetics of quantitative characters in relation to plant breeding. Indian Journal of Genetics 17: 318-328.

Pillay, M., Ogundiwin, E., Nwakanma, D.C., Ude, G. and Tenkouano, A. (2001). Analysis of genetic diversity and relationships in East African banana germplasm. Theoretical and Applied Genetics 102(6):965-970.

Price, N.S. (1995). In: Gowen, S. (ed.). The origin and development of banana and plantain cultivation. Chapman and Hall, London, UK. pp. 1-13.

Rekha, A.and Prasad, M.B.N.V. (1993). Genetic variability and character association in banana. Indian Journal of Horticulture 50: 36-40.

Robinson, H. F., Comstock, R.E. and Harvery, V. H. (1949). Estimates of heritability and degree of dominance in corn. Agronomy Journal 41: 353-359.
Sci. Technol. Arts Res. J., Oct-Dec 2014, 3(4): 19-28

SAS Institute. (2001). SAS software. SAS Institute INC., Cary. NC. USA.

Shepherd, K. (1957). Banana cultivars in East Africa. Tropical Agriculture 34: 277-286.

Silayoi, B., Wanichkul, K., Keawsompong, S., Saraduldhat, P. and Singhaburaudom, N. (1995). Study of mutation breeding of banana Kluai Khai. In: Final Report of an FAO/IAEA Co-ordinated Research Programme. Division of Nuclear Techniques in Food and Agriculture, Vienne, pp.65-77.

Simmonds, N.W. (1959). Bananas (1 $1^{\text {st }}$ edition). Longmans, Green and Co. Ltd., London, UK.

Singh, B. D. (1990). Plant Breeding. Kalyani Publishers, New Delhi, India.

Singh, R.K. and Chaudhry, B.D.(1985). Biometrical Methods in Quantitative Genetic Analysis. Kalynani Publishers, Ludhiana, India.

Sirisenaa, J.A. and Senanayake, S.G.J.N. (2000). Estimation of variability parameters within 'Mysore' banana clones and their implication for crop improvement. Scientia Horticulturae 84: 49-66.

Sivasubramanian, S. and Menon, M. (1973). Heterosis and inbreeding depression in rice. Madras Agricultural Journal 60: 1139.

Sneath, P.H., Sokal, R.R. (1973). Numerical Taxonomy. Freeman and Company, San Francisco,USA.

Sree Rangaswamy, S.R., Sambandamurthi, S. and Murugesan, M. (1980). In: Muthukrishnan, C.R., Abdul Khader, I.B.M.M.(eds.) Genetic analysis of banana., National Seminar on Banana Production Technology. Tamilnadu Agricultural University, Coimbatore, India. pp.50-56.

STATISTICA 7 Software. (2002). StatSoft Revision 06/03/03,U.S.A.

Stover, R.H. and Simmonds, N.W. (1987). Bananas (3 ${ }^{\text {rd }}$ edition). Longmans, London, UK.

Swennen R. and Vuylsteke, D.R. (1988). Bananas in Africa: Uses and prospects for improvement. Proceedings of an International Conference 17-20 October 1988, Ibadan, Nigeria. Crop Genetic resources for Africa Vol. II.

Swennen, R and Vuylsteke, D.R. (1987). In: Persley, G. J. and De Langhe, E.A. (eds.) Morphological taxonomy of plantain (Musa cultivars $A A B$ ) in West Africa. Banana and plantain breeding strategies: Proceedings of an international workshop held at Cairns, Australia, 13-17 Oct. 1986. ACIAR Proceeding 21:165-171.

Swennen, R., Vuylsteke, D. and Ortiz, R. (1995). Phenotypic diversity and patterns of variation in West and Central African plantains. Economic Botany 49: 320-327.

Tenkouano, A., Ortiz, R. and Baiyeri, K.P. (2002). Phenotypic and genetic correlations in Musa populations in Nigeria. African Crop Science Journal 10 (2): 121-132.

Vuylsteke, D., Ortiz, R. and Ferris, S. (1993). Genetic and agronomic improvement for sustainable production of plantain and banana in sub-Saharan Africa. African Crop Science Journal 1: 1-8. 